\title{
Diagnosis and management of endometriosis: summary of NICE guidance
}

This article (BMJ 2017;358:j3935, doi:10.1136/bmj.j3935) was initially published with incomplete authorship details: the list of authors should have included "on behalf of the Guideline
Committee." The members of the Guideline Committee are listed in the end matter of the article. 